\title{
Diagnóstico prenatal y resultado falso negativo por un caso de Síndrome de Down con cariotipo normal
}

\author{
Luis Arturo Lizcano-Gil MD, MSc; Katya Burgos BS; Diego Fernando Guarín MD.*
}

\section{RESUMEN}

Presentamos el caso de una mujer de 38 años remitida en la semana 29 de gestación a nuestro programa de diagnóstico prenatal, debido a que el estudio uItrasonográfico reveló retardo del crecimiento intrauterino, acortamiento de huesos largos, y polihidramnios. Se practicó y con características fenotípicas concordantes con el Síndrome de Down. Luego del nacimiento realizamos cariotipos en sangre y piel (este mostraron cariotipo normal 46, XY.

El Síndrome de Down con cariotipo normál es infrecuente, pero establece una rara limitación para el diagnóstico prenatal.

PALABRAS CLAVES: Cariotipo normal; diagnóstico prenatal; leucemia neonatal; resultado falso negativo; Síndrome de Down.

\section{SUMMARY}

We present the case of a 38 year-old woman attended in our prenatal diagnosis program due to fetal growth retardation, shortening of long bones, polyhydramnios. She underwent transabdominal amniocentesis at 29 weeks of gestation, and cytogenetic study was done, finding a normal karyotype 46, XY. At 32 weeks, a caesarean operation was performed because of acute fetal distress, obtaining a masculine newborn with phenotype features concordant to Down Syndrome. After the birth we did blood tests and also skin karyotypes (these last ones after his death). The samples were analyzed in our institution and in a reference laboratory. All the studies revealed a normal karyotipe 46, XY. Down Syndrome with normal karyotype is an infrequent situation, but it establishes a rare limitation of the prenatal diagnosis.

KEY WORDS: Prenatal diagnosis; Down Syndrome; normal karyotype; false negative result; neonatal leukemia.

\section{Introducción}

En la literatura médica se han descrito varios casos de pacientes con fenotipo concordante con Síndrome de Down, pero con cariotipo normal. Para explicar esta incongruencia, se ha propuesto la existencia de nuevos síndromes con fenotipo similar pero diferente etiología, fenocopias (2, 6-8), mosaicismos de bajo grado no identificables en tejidos fácilmente accesibles como sangre o piel y duplicaciones submicroscópicas del cromosoma 21 en la región q22 (1, 3-4, 9-10). Aunque el Síndrome de Down con cariotipo normal es raro, cuando se presenta un caso clínico como todos los descritos hasta el momento, es factible realizar estudios complementarios o proponer un diagnóstico diferencial. Sin embargo cuando el estudio es prenatal, se hace muy difícil suponer el diagnóstico de esta situación, aún en casos como éste, donde existía evidencia ecográfica sugestiva de una anormalidad cromosómica.

\section{Descripción del Caso}

Una mujer de 38 años fue remitida a nuestra institución cursando la semana 29 de su segunda gestación, debido a retardo del crecimiento intrauterino, acortamiento de huesos largos y polihidramnios sin malformaciones gastrointestinales. Se realizó amniocentesis y ecografía de detalle anatómico, con hallazgos similares a los ya descritos. La muestra se trató de acuerdo con los protocolos actuales de nuestro laboratorio (2 cultivos de líquido amniótico en frasco y 3 en slide chamber, técnica in situ). Siete días después se obtuvieron los cariotipos de los cultivos in situ, mostrando una constitución cromosómica normal (Cariotipo 46, XY. Bandeos GTG y QFQ, resolución de 450 bandas aproximadamente). Los resultados se informaron a los padres y al médico tratante.

A las 32 semanas de gestación se realizó cesárea indicada por sufrimiento fetal, obteniendo un recién nacido de

* División de Genética. Centro Colombiano de Fertilidad y Esterilidad, CECOLFES. Bogotá, Colombia. 
sexo masculino con un fenotipo concordante con el Síndrome de Down. Inicialmente se interpretó como un error de laboratorio. Uno de nosotros (Lizcano-Gil LA) examinó al recién nacido, quien tenía suficientes hallazgos fenotípicos para establecer el diagnóstico de Síndrome de Down (Tabla 1), incluyendo una leucemia neonatal, que explica el polihidramnios (12) y un defecto del septo interventricular.

\section{Tabla 1 INTERPRETACION DEL PUNTAJE SEGUN FRIED (5)}

6 a 8 signos: Se considera clínicamente confirmado. 3 a 5 signos: Se requiere estudio cromosómico con propósito diagnóstico. 0 a 2 signos: Se descarta el diagnóstico.

\begin{tabular}{|lc|}
\hline Signo & Presente \\
\hline Abundante piel en cuello & $\mathrm{Si}$ \\
Comisuras labiales inclinadas hacia abajo & $\mathrm{Si}$ \\
Hipotonía & $\mathrm{Si}$ \\
Fascies plana & $\mathrm{Si}$ \\
Displasia de pabellones auricularees & $\mathrm{No}$ \\
Pliegues epicánticos & $\mathrm{Si}$ \\
Espacio aumentado entre primero y segundo artejos & $\mathrm{Si}$ \\
Protrusión lingual & $\mathrm{Si}$ \\
\hline
\end{tabular}

Realizamos estudios en sangre luego del parto y en piel después de su fallecimiento. Se procesaron cultivos simultáneos en nuestro laboratorio y en otro de referencia y siempre se encontraron cariotipos normales. No fue posible realizar estudios moleculares. El cariotipo de ambos padres es normal. No obtuvimos consentimiento de los padres para presentar las fotos del paciente, quien murió a los 7 días de edad.

\section{Discusión}

El Síndrome de Down con cariotipo normal es una situación infrecuente, pero representa un factor limitante para el diagnóstico prenatal.

Aunque no se realizaron estudios moleculares debido a que no estaban disponibles en nuestro medio, la evaluación de los hallazgos fenotípicos del paciente de acuerdo con la aproximación diagnóstica empleada por Fried (5), quien estudió 19.000 recién nacidos sin obtener resultados falsos positivos o falsos negativos, el tipo de cardiopatía documentada y la coexistencia de leucemia de inicio prenatal que se considera como una condición hematológica rara, más frecuentemente encontrada en pacientes con este diagnóstico (11), nos permiten suponer con un mínimo margen de error que este paciente presentaba Síndrome de Down.

\section{BIBLIOGRAFIA}

1. Ahlbom B E, Goetz P, Korenberg J R, Pettersson U, Seemanova E, Wadelius C, Zech L, Anneren G. Molecular analysis of chromosome 21 in a patient with a phenotype of Down syndrome and apparently normal karyotype. Am J Med Genet 1996; 63(4): 566-572.

2. Bowen p, Chernick B C, Campbell B J, Rouget A. Mild characteristic of Down syndrome with normal Karyotype in cultured Iymphocytes and skin fibroblast. Birth Defects Orig Artic Ser 1974; 10(10): 43-48.

3. Delabar J M, Sinet PM, Chadefaux B, Nicole A, Gegonne A, Stehelin D, Fridlansky F, Creau-Goldberg N, Turleau C, de Grouchy J. Submicroscopic duplication of chromosome 21 and trisomy 21 phenotype (Down syndrome). Hum Genet 1987; 76(3): 225-229.

4. Delabar J M, Theophile D, Rahmani Z, Chettouh Z, Blouin JL, Prieur M, Noel B, Sinet PM. Molecular rnapping of twenty-four features of Down syndrome on chromosome 21. Eur J Hum Genet 1993; 1(2); 114-124.

5. Fried K. A score based on eight signs in the diagnosis of Down syndrome in the newborn. J Ment Defic Res 1980; 24(3): 181-185.

6. Gale A N, Lacassie Y, Rogers J G, Levin LS, McKusick VA. Two "new" autosomal recessive mental retardation syndromes observed among the Amish. Birth Defects Orig Artic Ser 1977; 13(3B): 127-138.

7. Gripp K W, Nicholson L, Scott C 1 Jr. Apparently new syndrome of congenital cataracts, sensorineural deafness, Down syndrome-like facial appearance, short stature, and mental retardation. Am J Med Genet 1996; 61(4): 382-386.

8. Hall B. Down's syndrome (mongolism) with normal chromosomes. Lancet 1962; 2: 1026-1027.

9. Korenberg J R, Kawashirna H, Pulst SM, Ikeuchi T, Ogasawara N, Yamamoto K, Schonberg SA, West R, Allen L, Magenis E, et al. Molecular definition of a region of chromosome 21 that causes features of the Down syndrome phenotype. Am J Hum Genet 1990; 47(2): 236-246.

10. McCormick M K, Schinzel A, Petersen M B, Stetten G, Driscoll DJ, Cantu ES, Tranebjaerg L, Mikkelsen M, Watkins PC, Antonarakis SE. Molecular genetic approach to the characterization of the "Down syndrome region" of chromosome 21. Genomics 1989; 5(2): 325-331.

11. Zipursky A, Poon A, Doyle J. Leukemia in Down syndrome: a review. Pediatr Hematol Oncol 1992; 9(2): 139-149.

12. Zipursky A, Rose T, Skidmore M, Thorner P, Doyle J. Hydrops fetalis and neonatal leukemia in Down syndrome. Pediatr Hematol Oncol 1996; 13(1): $81-87$. 